\title{
CARACTERIZACIÓN DE NANOPARTÍCULAS DE ZnO SINTETIZADAS POR EL MÉTODO DE PRECIPITACIÓN Y SU EVALUACIÓN EN LA INCORPORACIÓN EN PINTURAS ESMALTE
}

\author{
Pablo Aquino*a, Ana María Osorio, Efraín Ninán ${ }^{\mathrm{a}}$, Fernando Torres ${ }^{\mathrm{b}}$
}

\begin{abstract}
RESUMEN
El objetivo del presente estudio consiste en determinar con diversas técnicas experimentales, como TEM, XRD y DLS, el tamaño, la morfología y algunas características propias de las nanopartículas de $\mathrm{ZnO}$ sintetizadas por el método de la precipitación. Las nanopartículas sintetizadas fueron incorporadas en muestras de pintura esmalte y su evaluación por FTIR y GIXRD determinaron que existe compatibilidad entre ambos materiales. Además, se evaluó la resistencia a la degradación y el comportamiento de curado del composito a través de ensayos térmicos, como TGA y DSC.
\end{abstract}

Palabras clave: Caracterización nanopartículas $\mathrm{ZnO}$, síntesis nanopartículas de $\mathrm{ZnO}$ por el método de precipitación, evaluación compatibilidad NPs-ZnO con pintura esmalte.

\section{CHARACTERIZATION OF ZnO NANOPARTICLES SYNTHESIZED BY PRECIPITATION METHOD AND ITS EVALUATION IN THE INCORPORATION IN ENAMEL PAINTS}

\begin{abstract}
The objective of the present study is to determine the size, morphology and some characteristics of the $\mathrm{ZnO}$ nanoparticles synthesized by precipitation method using various experimental techniques, such as TEM, XRD and DLS. The synthesized nanoparticles were incorporated in samples of enamel paint and their evaluation by FTIR and GIXRD determined that there is compatibility with both materials. Also the degradation resistance and curing behavior of the composite were evaluated through thermal tests, such as TGA and DSC.
\end{abstract}

Key words: Characterization of $\mathrm{ZnO}$ nanoparticles, synthesis of $\mathrm{ZnO}$ nanoparticles by precipitation method, evaluation NPs-ZnO compatibility with enamel paint.

\footnotetext{
a Laboratorio de Nanotecnología e Innovación Tecnológica de la FQIQ - UNMSM 140291pablo@gmail.com

b Laboratorio de Polímeros y Bionanomateriales de la sección de Ingeniería Mecánica - PUCP
} 


\section{INTRODUCCIÓN}

Las pinturas anticorrosivas representan una barrera de protección de materiales como los metales frente a casos de corrosión. La baja durabilidad, y disminución del efecto anticorrosivo generan grandes pérdidas económicas en los sectores involucrados, pero si bien el proceso de corrosión es inevitable al ser un proceso natural, lo que se pretende es controlar su velocidad, a partir de compuestos a nivel nanométrico que permitan retrasar su efecto en el material ${ }^{1}$.

La aplicación de las nanopartículas a los recubrimientos ha sido objeto de estudios dirigidos a la producción de pinturas con diferente composición química y con diferentes aplicaciones ${ }^{2}$.

Las nanopartículas de óxido de zinc ( $\mathrm{NPs}-\mathrm{ZnO})$ pueden presentar diferentes comportamientos a causa de una mayor energía y área superficial, así como una banda prohibida, $\mathrm{Eg}^{3}$, variable. Esta última propiedad permite las aplicaciones en diferentes áreas como optoelectrónica, cosméticos, textil ${ }^{4}$, catalizadores, cerámicos, películas finas ${ }^{5}$, sensores de gas, aditivos de caucho, pigmentos ${ }^{6}$, transistores y transductores ${ }^{7}$.

En la literatura encontramos varios métodos adaptados para la preparación de NPs-ZnO como el método sol-gel ${ }^{8}$, síntesis por precipitación ${ }^{9-12}$, la síntesis química húmeda y química verde ${ }^{13}$, método hidrotermal ${ }^{14}$, entre otros.

En los trabajos mencionados ${ }^{8-12}$ se emplean técnicas variadas de caracterización de NPsZnO como DR-X, UV-Visible, IR-TF, TEM, SEM, entre otros, para la determinación de la cristalización, tamaño y morfología. Por otro lado, la incorporación de NPs-ZnO en diversos tipos de pinturas está cobrando mucho interés por las propiedades mejoradas respecto a las convencionales ${ }^{15}$. Por otro lado la resistencia a la degradación térmica y el comportamiento de curado son realizados a través de ensayos térmicos, como TGA y DSC.

\section{PARTE EXPERIMENTAL}

En este trabajo se ha realizado la síntesis de NPs-ZnO a través del método de precipitación, empleando como precursor $\mathrm{Zn}\left(\mathrm{CH}_{3} \mathrm{COO}\right)_{2}$ (q.p), en medio acuoso a una temperatura de reacción de $50^{\circ} \mathrm{C}$. El proceso de síntesis se realizó por goteo de la solución de $\mathrm{Zn}\left(\mathrm{CH}_{3} \mathrm{COO}\right)_{2}$ en una solución de $\mathrm{NaOH}$ por un tiempo de 2 horas y 20 minutos. Luego se procedió a la filtración y calcinación como se muestra en el esquema 1. 


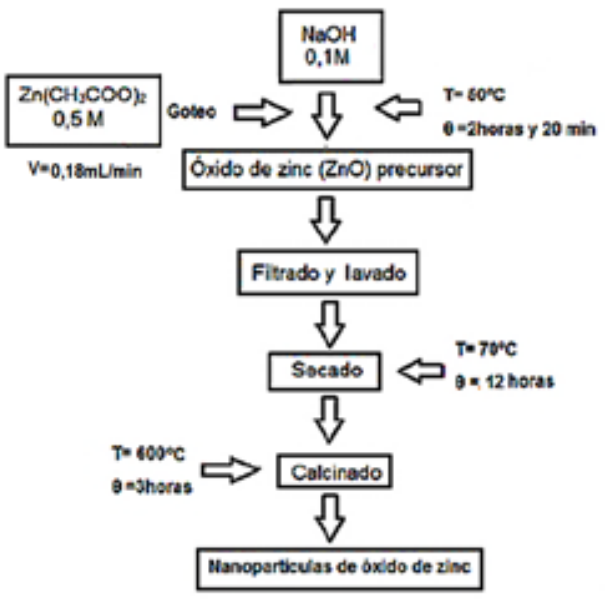

Esquema 1. Procedimiento sintético de las NPs-ZnO

Después del proceso de síntesis se obtuvo un rendimiento de reacción de $64 \%$. La caracterización del grado de cristalización de las NPs-ZnO fue por difracción de rayos $\mathrm{X}$ (XRD), el tamaño por dispersión dinámica de Luz (DLS), el tamaño y morfología simultáneamente fue realizada por microscopía electrónica de transmisión (TEM).

La incorporación de las NPs-ZnO en la pintura esmalte fueron en porcentajes de 0,1 a $5,0 \% \mathrm{w}$ y se caracterizaron por espectroscopía infrarroja con transformada de Fourier (FTIR) y difracción de rayos $\mathrm{X}$ de incidencia rasante (GIXRD). Además se realizaron ensayos térmicos para evaluar la resistencia a la degradación térmica y su comportamiento de curado por análisis termogravimétrico (TGA) y calorimetría diferencial de barrido (DSC), respectivamente.

\section{RESULTADOS Y DISCUSIÓN}

\section{Síntesis de las nanopartículas de óxido de zinc}

En el esquema 2 se muestran las reacciones de la síntesis de las NPs-ZnO, la cual se llevó a cabo siguiendo el mecanismo de precipitación, que fueron reportado por $\mathrm{Wu} \mathrm{C.}{ }^{15}$ quien explica que el $\mathrm{Zn}\left(\mathrm{CH}_{3} \mathrm{COO}\right)_{2}$ puede convertirse en $\mathrm{Zn}(\mathrm{OH})_{2}$ coloidal bajo una solución alcalina ( $\mathrm{pH}=13-14)$, como se muestra en la reacción (1). Durante el proceso hidrotermal, parte del $\mathrm{Zn}(\mathrm{OH})_{2}$ coloidal se disocia a $\mathrm{Zn}^{2+}$ y $\mathrm{OH}^{-}$de acorde a la reacción (3). Cuando la concentración de $\mathrm{Zn}^{2+}$ y $\mathrm{OH}^{-}$alcanzan el grado de súper saturación, se forman núcleos de $\mathrm{ZnO}$ de acuerdo a la reacción (4). 


$$
\begin{aligned}
& \mathrm{Zn}\left(\mathrm{CH}_{3} \mathrm{COO}\right)_{2(a \mathrm{cc})}+2 \mathrm{NaOH}_{(\mathrm{acc})} \stackrel{50{ }^{\circ \mathrm{C}}}{\rightleftarrows} \mathrm{Zn}(\mathrm{OH})_{2(\mathrm{gel})}+2 \mathrm{CH}_{3} \mathrm{COONa}_{(a c)} \quad p H=13-14 \mathrm{Rx}(1) \\
& \mathrm{Zn}(\mathrm{OH})_{2(\mathrm{gel})}+2 \mathrm{H}_{2} \mathrm{O} \stackrel{500^{\circ} \mathrm{C}}{\rightleftarrows} \mathrm{Zn}^{2+}+2(\mathrm{OH})^{-}+2 \mathrm{H}_{2} \mathrm{O} \quad \mathrm{Rx}(2) \\
& \mathrm{Zn}^{2+}+2(\mathrm{OH})^{-}+2 \mathrm{H}_{2} \mathrm{O} \stackrel{50 * \mathrm{C}}{\rightleftarrows} \mathrm{Zn}(\mathrm{OH})_{4}^{2-}+2 \mathrm{H}^{+} \quad \mathrm{Rx}(3) \\
& \mathrm{Zn}(\mathrm{OH})_{4}^{2-} \stackrel{50^{\circ} \mathrm{C}}{\rightleftarrows} \mathrm{ZnO}+\mathrm{H}_{2} \mathrm{O}+2(\mathrm{OH})^{-} \mathrm{pH}=7-8 \quad \mathrm{Rx}(4)
\end{aligned}
$$

Esquema 2. Etapas de síntesis de NPs-ZnO por el método de precipitación ${ }^{15}$

\section{Caracterización de las nanopartículas de óxido de zinc}

\section{Difracción de Rayos-X (XRD)}

La figura 1 muestra el espectro de difracción de rayos X de las NPs-ZnO. El espectro muestra picos bien definidos a $2 \theta=31^{\circ}, 34^{\circ}, 36^{\circ}, 48^{\circ}, 56^{\circ}, 63^{\circ}$ y $68^{\circ}$, respectivamente, que eran debido a las difracciones de los planos (100), (002), (101), (102), (110), (103) y (112) típico de ZnO en la estructura cristalina tipo Wurtzita. El tamaño medio del cristalito de $\mathrm{ZnO}$ se muestra en la tabla 1, el cual se estimó por comparación tomando dos de los máximos picos de difracción de un patrón de $\mathrm{LaB}_{6} \mathrm{y}$ de acuerdo con la ecuación de Scherrer, el tamaño del cristalito se encuentra entre 72 y $81 \mathrm{~nm}$ aplicado a los picos principales $37,48^{\circ}$ y $30,42^{\circ}$, respectivamente.

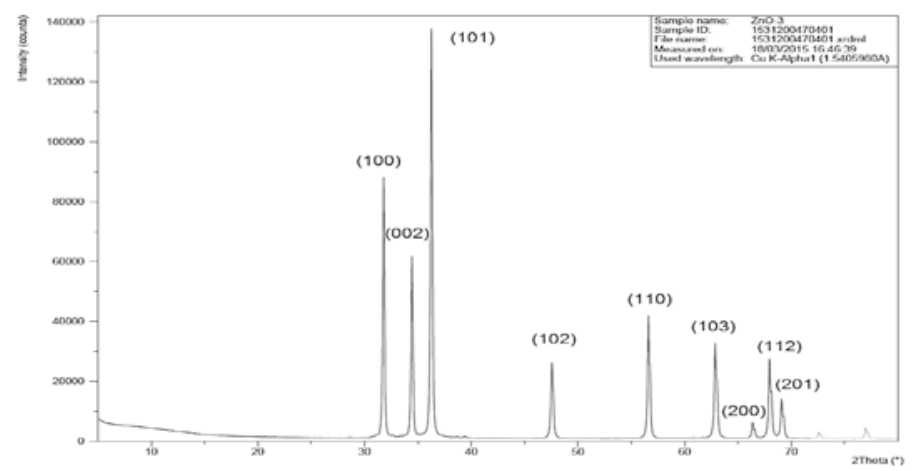

Figura 1. Difractograma de rayos $\mathrm{X}$ de las NPs-ZnO

Tabla 1. Tamaño de los cristalitos de $\mathrm{ZnO}$

\begin{tabular}{|l|r|r|r|r|r|}
\hline Muestra & B obs. [ $\left.{ }^{\circ} 20\right]$ & B std. ['20] & Peak pos. [ $\left.{ }^{\circ} 20\right]$ & B struct. [ $\left.{ }^{\circ} 20\right]$ & Crystallite size[Å] \\
\hline 1531200470401 & 0.160 & 0.058 & 31.768 & 0.102 & 810 \\
\hline 1531200470401 & 0.168 & 0.052 & 36.254 & 0.116 & 721 \\
\hline
\end{tabular}




\section{Dispersión dinámica de luz (DLS)}

El tamaño hidrodinámico de las NPs-ZnO se determinó utilizando analizador de tamaño de partícula DLS se muestran en la figura 2. El histograma de intensidad (figura 2a), volumen (figura 2b) y la distribución del número (figura 2c), muestran el tamaño de las nanopartículas que poseen un tamaño medio de $88,3 \pm 0,62 \mathrm{~nm}$, tabla 2 .

El histograma de la distribución de intensidad muestra el pico máximo de la aglomeración debido interacciones intermoleculares débiles, los cuales fue confirmada también por el histograma de distribución del volumen. La función de autocorrelación presenta una sola pendiente. Esta nos da información sobre la monodipersión que da la distribución que presentan las NPs-ZnO.

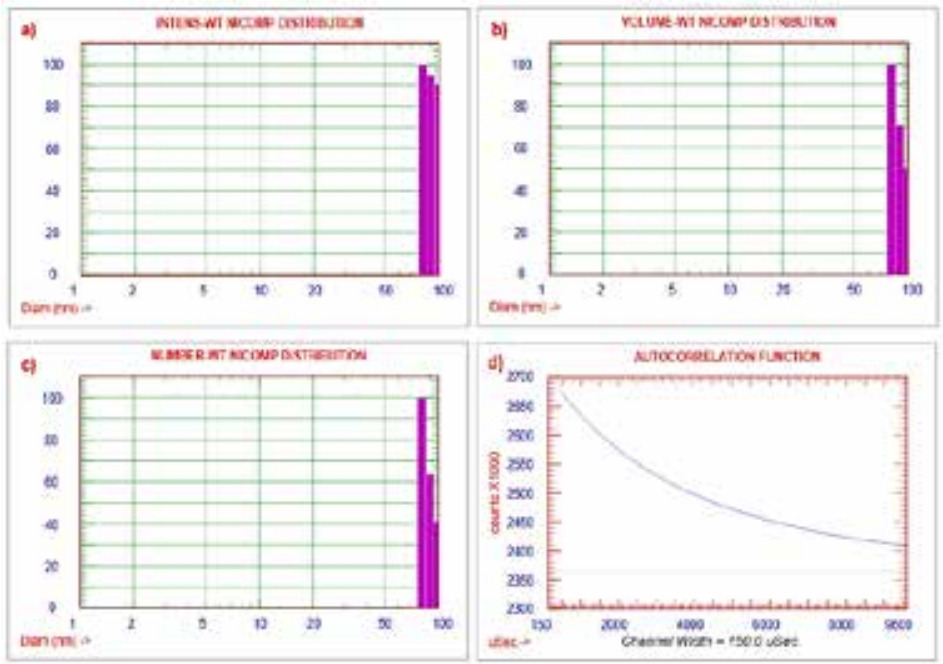

Figura 2. Histograma de la distribución del tamaño de partícula obtenida por DLS (a) distribución de la intensidad, (b) la distribución del volumen, (c) la distribución de número y (d) la función de correlación

Tabla 2. Tamaño hidrodinámico de las NPs-ZnO

\begin{tabular}{|c|c|c|c|}
\hline Pico & $\begin{array}{c}\text { Medida del } \\
\text { Diámetro }(\mathbf{n m})\end{array}$ & $\begin{array}{c}\text { Desviación } \\
\text { Estándar }(\mathbf{n m})\end{array}$ & \%Volumen \\
\hline 1 & 88,3 & 0,62 & 100 \\
\hline
\end{tabular}




\section{Microscopía Electrónica de Transmisión (TEM)}

En la micrografía TEM de las NPs-ZnO (figura 3a) se puede observar una distribución homogénea en la escala de medición de $100 \mathrm{~nm}^{11}$. Las nanopartículas presentan una morfología semi-hexagonal y una amplificación superior en la micrografía HR-TEM (figura 3 b), es compatible con la naturaleza cristalina del $\mathrm{ZnO}$ nanopartícula en el plano basal de la estructura tipo wurtzita ${ }^{3,4}$. La distribución del tamaño de partícula se ha calculado mediante

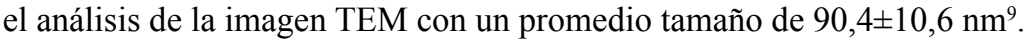

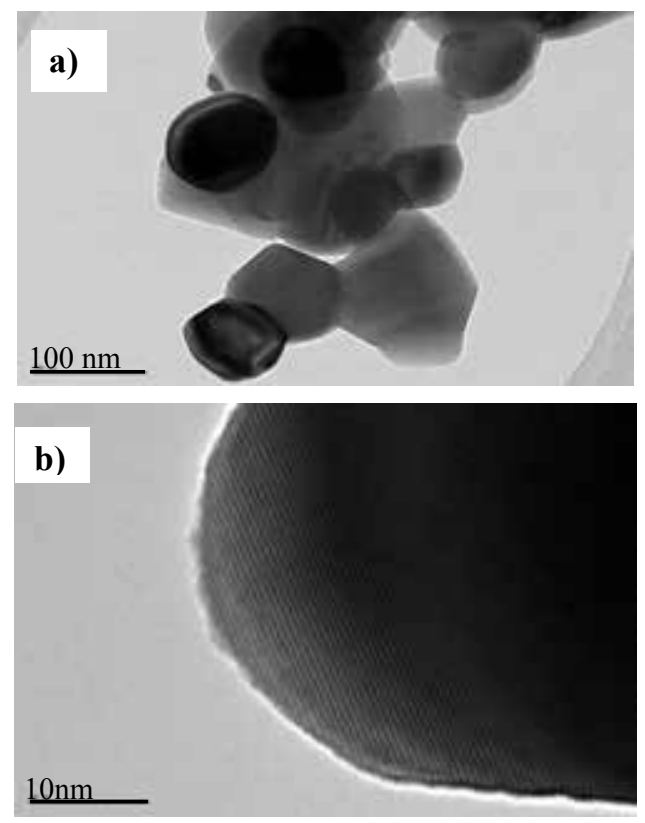

Figura 3. Imagen TEM de NPs-ZnO obtenidas por el método de precipitación (a) y de alta resolución de un plano de la estructura tipo wurtzita (b)

Las medición del tamaño de NPs-ZnO por DLS y por TEM, reportan tamaños similares, se puede decir que son técnicas que pueden emplearse para corrobar que el tamaño de los cristalitos obtenidos por el método de la precipitación nos permiten obtener nanopartículas, las cuáles debido a sus propiedades excepcionales pueden tener diversas aplicaciones. La variación del tamaño medidos por XRD $(72-81 \mathrm{~nm})$ no resulta muy precisa debido a la variación de las condiciones experimentales de medición, pero es muy valioso en la determinación del sistema de cristalización, tal como es en nuestro caso, las NPs-ZnO pertenecen al sistema cristalino hexagonal correspondiente a una estructura tipo wurzita.

\section{Tratamiento e incorporación de las nanopartículas}

Las NPs-ZnO se dispersaron en la matriz de la pintura esmalte en cantidades de 0,$1 ; 0,5$; 1,0 y $5,0 \% \mathrm{w}$ como se muestra en la figura 4 . Se llevó a cabo el análisis superficial de la 
capa de pintura esmalte con y sin NPs-ZnO a través de fotografías y se ha observado que las nanopartículas en 0,1 y $0,5 \%$ w no efectuaron cambios significativos en la superficie de la capa de pintura, mientras que para 1,0 y $5,0 \% \mathrm{w}$, estas nanopartículas se aglomeran en la superficie. La coloración de la pintura no sufrió ningún cambio, ya que las partículas menores $100 \mathrm{~nm}$ pueden aumentar la transparencia de la película en la región de longitud de onda visible de luz.

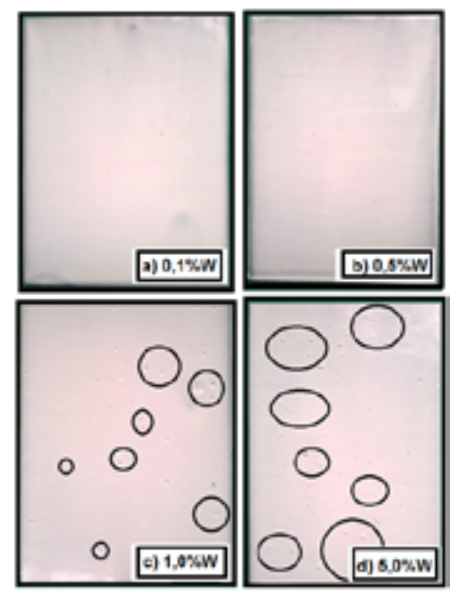

Figura 4. Análisis superficial de las capas de pintura esmalte con NPs-ZnO dispersadas en proporciones: a) $0,1 \% \mathrm{w}$, b) $0,5 \% \mathrm{w}$, c) $1,0 \% \mathrm{w}$ y d) $5,0 \% \mathrm{w}$

\section{Evaluación la incorporación de las nanopartículas en la pintura esmalte por FT-IR}

Espectroscopia FT-IR se utilizó para evaluar si las nanopartículas se encuentran presente sobre la superficie de la película de pintura. La figura 5 muestra la superposición de espectros de las NPs-ZnO (a), de la pintura (b) y de una de las muestras $0,5 \%$ de NPs-ZnO en pintura (c).

En el espectro (a), el pico que se observa a partir de $400 \mathrm{~cm}^{-1}-500 \mathrm{~cm}^{-1}$ es debido a vibraciones de las nanopartículas de $\mathrm{Zn}-\mathrm{O}$, mencionado anteriormente.

El espectro (b) de la pintura esmalte, al tener como componente principal a la resina alquídica, presentan una banda de absorción entre los 3390 - $3232 \mathrm{~cm}^{-1}$ que representa al grupo OH. Las bandas 2927 y $2854 \mathrm{~cm}^{-1}$, corresponden a alcanos mientras que a $1724 \mathrm{~cm}^{-1}$ indica la presencia de ésteres (tensión $\mathrm{C}=\mathrm{O}$ ) y a $1265 \mathrm{~cm}^{-1}$ tensión $\mathrm{C}-\mathrm{O}-\mathrm{C}$. La banda de adsorción entre 550$450 \mathrm{~cm}^{-1}$ corresponde a los alcanos de cadena larga. Lo mismo se puede decir de las bandas de absorción del espectro del composito pintura-NPs-ZnO (c), pero existe una diferencia en el \% $\mathrm{T}$, debido a la dispersión homogénea de las nanopartículas sobre la matriz polimérica y la formación de interacciones intermoleculares entre los grupos carboxílicos de la resina alquídica y las NPs-ZnO. 


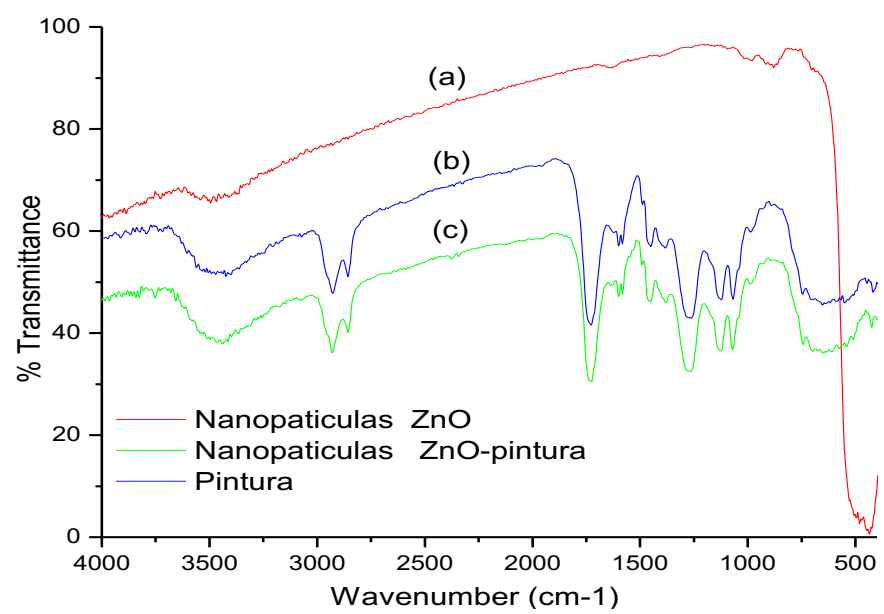

Figura 5. Espectro FTIR del composito, NPs-ZnO dispersas en la pintura esmalte

Tabla 3. Datos obtenidos del espectro de la pintura y pintura-NPs-ZnO

\begin{tabular}{|c|c|c|c|}
\hline \multicolumn{2}{|c|}{ Pintura } & \multicolumn{2}{|c|}{ Pintura-NPs $\mathrm{ZnO}$} \\
\hline $\begin{array}{c}\text { Grupo } \\
\text { Funcional }\end{array}$ & Wavenumber $\left(\mathrm{cm}^{-1}\right)$ & $\begin{array}{c}\text { Grupo } \\
\text { Funcional }\end{array}$ & Wavenumber $\left(\mathrm{cm}^{-1}\right)$ \\
\hline$(\mathrm{OH})^{-}$ & 3432 & $(\mathrm{OH})^{-}$ & 3448 \\
\hline \multirow{2}{*}{ alcanos } & 2927 & \multirow{2}{*}{ alcanos } & 2927 \\
\hline & 2854 & & 2854 \\
\hline tensión $\mathrm{C}=\mathrm{O}$ & 1724 & tensión $\mathrm{C}=\mathrm{O}$ & 1724 \\
\hline tensión $\mathrm{C}-\mathrm{O}-\mathrm{C}$ & 1265 & tensión $\mathrm{C}-\mathrm{O}-\mathrm{C}$ & 1265 \\
\hline \multirow{2}{*}{$\begin{array}{c}\text { alcanos de } \\
\text { cadena larga }\end{array}$} & 516 & \multirow{2}{*}{$\begin{array}{l}\text { alcanos de } \\
\text { cadena larga }\end{array}$} & 640 \\
\hline & 466 & & 470 \\
\hline
\end{tabular}

\section{Evaluación por Difracción de Rayos X de Incidencia Rasante (GIXRD)}

Con el fin de obtener una mejor comprensión de la estructura de la capa (pintura-NPs-ZnO) y ver si la incorporación de las nanopartículas se encuentra en la superficie de la capa, debajo o entre el soporte metálico y la capa de pintura, se realizaron estudios de difracción de incidencia rasante (GIXRD).

La figura 4 muestra el difractograma de la capa (pintura- $0,5 \% \mathrm{wNps} \mathrm{ZnO}$ ) a un ángulo de incidencia de $\theta=0^{\circ}$ y por análisis de fases, se observan los componentes de la pintura (tabla 6). Mientras que en la figura 7, a un ángulo de incidencia $\theta=5^{\circ}$, se puede observar algunos picos característicos del $\mathrm{ZnO}$ (tabla 5), además de algunos componentes de la pintura y sustrato metálico ${ }^{16-18}$. De acuerdo a este estudio las nanopartículas de óxido de zinc se encuentran en la superficie de la película de pintura. 


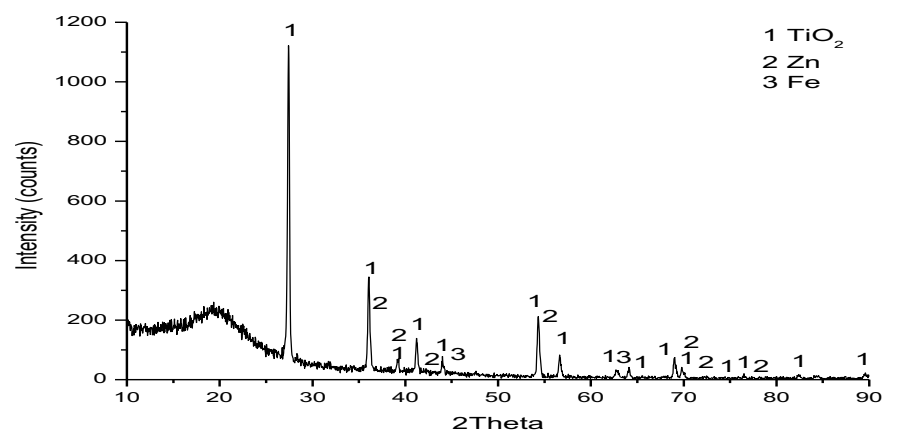

Figura 6. Difractograma por incidencia rasante de la capa (pintura- $0,5 \% \mathrm{wNPs} \mathrm{ZnO}$ ) a un ángulo de incidencia de $0^{\circ}$.

Tabla 4. Análisis de fases de Difracción de Rayos-X de Incidencia Rasante $\left(\theta=0^{\circ}\right)$, de la capa (pintura-0,5\%wNPs NPs $\mathrm{ZnO}$ )

\begin{tabular}{cccll}
\hline Visible & Code & Ref. Code & $\begin{array}{c}\text { Compound } \\
\text { Name }\end{array}$ & $\begin{array}{c}\text { Chemical } \\
\text { Formula }\end{array}$ \\
\hline$*$ & 1 & $01-072-1148$ & Rutile & $\mathrm{Ti} \mathrm{O} 2$ \\
$*$ & 2 & $03-065-3358$ & Zinc & $\mathrm{Zn}$ \\
$*$ & 3 & $00-006-0696$ & Iron, syn & $\mathrm{Fe}$ \\
\hline
\end{tabular}

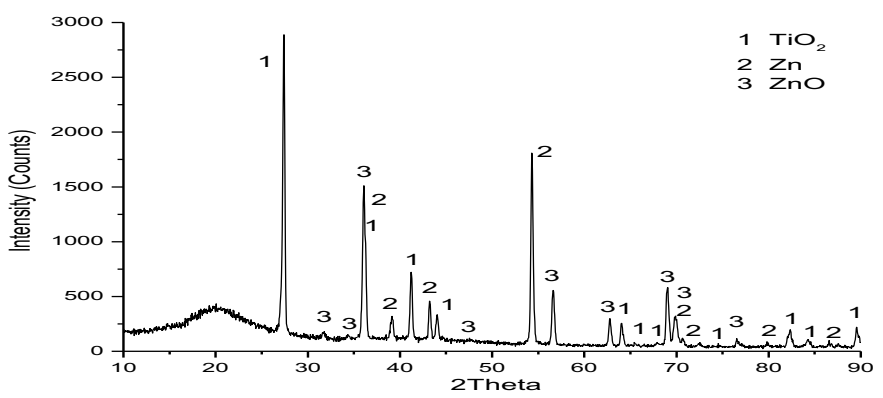

Figura 7. Difractograma por incidencia rasante de la capa (pintura- $0,5 \% \mathrm{wNPs}$ NPs $\mathrm{ZnO}$ ) a un ángulo de incidencia de $5^{\circ}$.

Tabla 5. Análisis de fases de Difracción de Rayos- $X$ de Incidencia Rasante $\left(\theta=5^{\circ}\right)$, de la capa (pintura-0,5\%wNPs NPs $\mathrm{ZnO}$ )

\begin{tabular}{ccrll}
\hline Visible & Code & Ref. Code & $\begin{array}{c}\text { Compound } \\
\text { Name }\end{array}$ & $\begin{array}{c}\text { Chemical } \\
\text { Formula }\end{array}$ \\
\hline$*$ & 1 & $01-072-1148$ & Rutile & $\mathrm{Ti} \mathrm{O} 2$ \\
$*$ & 2 & $03-065-3358$ & Zinc & $\mathrm{Zn}$ \\
$*$ & 3 & $01-070-2551$ & Zincite, syn & $\mathrm{Zn} \mathrm{O}$ \\
\hline
\end{tabular}




\section{Ensayos térmicos del composito pintura-NPs-ZnO}

Se evaluó la resistencia a la degradación térmica y el comportamiento de curado de los compositos pintura- $0,1 \%$ wNPs-ZnO y pintura- $0,5 \%$ wNPs-ZnO.

\section{Análisis Termogravimétrico (TGA)}

Se utilizó el análisis termogravimétrico (TGA) para examinar la estabilidad térmica de la pintura esmalte y de los compositos pintura- $0,1 \% \mathrm{wNPs}-\mathrm{ZnO}$ y pintura- $0,5 \% \mathrm{wNPs}-\mathrm{ZnO}$. En la figura 8 se observa la curva termogravimétrica tomada de la pintura esmalte, el cual presenta una pérdida de peso inicial aproximadamente de $0,86 \%$ a una temperatura entre $100-150^{\circ} \mathrm{C}$, debido a la pérdida de humedad. En el caso de la pintura esmalte con $0,1 \% \mathrm{y}$ $0,5 \% \mathrm{~W}$ de NPs-ZnO, los resultados indicaron pérdidas de humedad de $1,19 \%$ y $0,84 \% \mathrm{~W}$, respectivamente.

Las segundas pérdidas de peso se llevaron a cabo en el intervalo de temperaturas entre 200 y $600{ }^{\circ} \mathrm{C}$, donde la pérdida de peso después de 300 corresponde a la descomposición del polímero de la pintura esmalte.

En comparación con los compositos, la descomposición del polímero para pintura- $0,1 \%$ NPs-ZnO se mantiene similar, mientras que la descomposición del polímero se redujo en aproximadamente en 5,0 \% para el caso de pintura-0,5\%-NPs-ZnO, esta reducción está relacionada con la interacción fuerte en la interfaz de las NPs-ZnO y el material polimérico de la pintura ${ }^{19}$.

Las pérdidas totales para la pintura esmalte de $52,34 \% \mathrm{~W}$, mientras $54,61 \% \mathrm{~W}$ y $49,17 \% \mathrm{~W}$ para los compositos con $0,1 \mathrm{~W} \%$ y $0,5 \% \mathrm{~W}$ de $\mathrm{NPs}-\mathrm{ZnO}$, respectivamente.

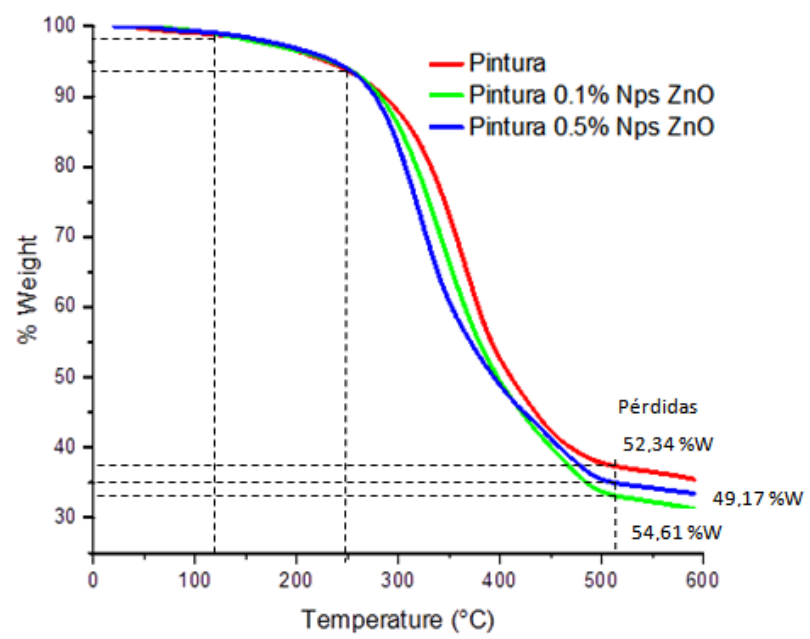

Figura 8. Análisis termogravimétrico (TGA) de pintura, pintura con $0,1 \% \mathrm{~W}$ NPs $\mathrm{ZnO}$ y pintura con $0,5 \% \mathrm{~W}$ NPs $\mathrm{ZnO}$ 


\section{Calorimetría Diferencial de Barrido (DSC)}

Se empleó calorimetría diferencia de barrido para estudiar el comportamiento de curado de la pintura esmalte y de los compositos de pintura- $0,1 \% \mathrm{NPs}-\mathrm{ZnO}$ y pintura- $0,5 \% \mathrm{NPs}-\mathrm{ZnO}$. Los termogramas DSC se muestran en la figura 9 y los valores de entalpía de curado de las muestras se observaron a partir de los diagramas DSC (tabla 6), donde los primeros picos endotérmicos, por debajo de $100{ }^{\circ} \mathrm{C}$, están asociados a la evaporación del agua. Los picos que caen en el intervalo de temperatura de 250-350 ${ }^{\circ} \mathrm{C}$ están relacionados con la entalpia de curado $^{20}$.

La entalpía de curado de la pintura esmalte disminuye de $23,12 \mathrm{~mW}$ a $21,69 \mathrm{~mW}$ y a 20,96 $\mathrm{mW}$ para los compositos de pintura con $0,1 \% \mathrm{w}$ y $0,5 \% \mathrm{w}$ en $\mathrm{NPs}-\mathrm{ZnO}$, respectivamente. Esta disminución de la entalpía está relacionada con el impedimento estérico y las interacciones físicas entre revestimiento y nanopartículas que conducen a aumento de la viscosidad de la pintura antes del curado. También las nanopartículas pueden reducir la densidad de reticulación del revestimiento al afectar a su comportamiento de curado ${ }^{21}$.

Tabla 6. Valores de la entalpía de curado de la pintura esmalte con 0,$0 ; 0,1 ; 0,5 \% \mathrm{~W}$ de NPs$\mathrm{ZnO}$ obtenidas a partir termogramas de DSC.

\begin{tabular}{|c|c|c|}
\hline \multirow{2}{*}{ Muestra } & Pico 1 & Pico 2 \\
\cline { 2 - 3 } & $\begin{array}{c}\text { Heat Flow } \\
(\mathrm{mW})\end{array}$ & $\begin{array}{c}\text { Heat Flow } \\
(\mathrm{mW})\end{array}$ \\
\hline 0 NPs ZnO & 21,24 & 23,12 \\
\hline $0,1 \% \mathrm{NPs} \mathrm{ZnO}$ & 20,78 & 21,69 \\
\hline $0,5 \%$ NPs ZnO & 20,36 & 20,96 \\
\hline
\end{tabular}

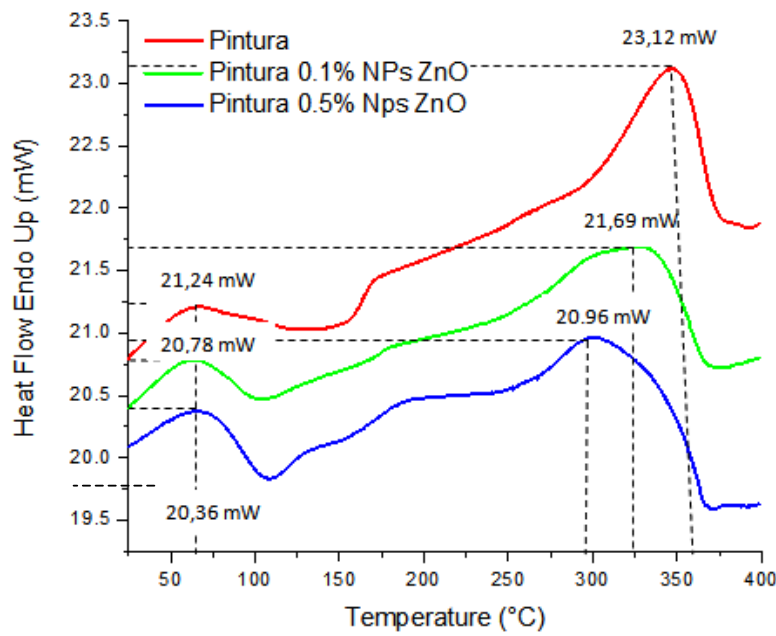

Figura 9. Termograma de la pintura esmalte, pintura con $0,1 \% \mathrm{NPs} \mathrm{ZnO}$ y pintura con $0,5 \% \mathrm{NPs}$ $\mathrm{ZnO}$ empleando calorimetría diferencial de barrido (DSC) 


\section{CONCLUSIONES}

- Se han sintetizado NPs-ZnO con un tamaño promedio de 90,4 nm, con diámetro hidrodinámico de $88,3 \mathrm{~nm}$ y estructura tipo wurtzita, a partir del método de precipitación, utilizando el acetato de zinc como precursor a $50^{\circ} \mathrm{C}$ con un rendimiento de $64 \%$.

- Las mezclas de pintura esmalte alquídica con NPs-ZnO al 0,1\% y 0,5\%w presentan mejor apariencia respecto a las formuladas con 1,0 y 5,0 \% de acuerdo al estudio de las fotografías.

- La evaluación de la incorporación NPs-ZnO se demostró en forma comparativa por la técnica instrumental FTIR, a través de la presencia de la banda entre $500-400 \mathrm{~cm}-1$, que es debido al enlace $\mathrm{Zn}-\mathrm{O}$ y a las bandas características de la resina alquídica de la pintura esmalte, que son compatibles, y determinan un sistema de dispersión estable. La evaluación por GIXRD indicó que las NPs-ZnO se encuentran en la superficie de la pintura.

- Los ensayos térmicos por TGA indicaron que se obtuvo una reducción de $0,4 \%$ y $5,0 \%$ en la descomposición del polímero de la pintura con 0,1 y $0,5 \%$ de $\mathrm{Nps}-\mathrm{ZnO}$, respectivamente, originando un material de mayor resistencia a la descomposición térmica.

- Se redujo la entalpía de curado de la pintura esmalte en 6,20 y 9,40 \% con adición de 0,1 y $0,5 \%$ de NPs-ZnO, respectivamente, formulando así una pintura con mayor velocidad de secado, con respecto a la tradicional.

\section{AGRADECIMIENTO}

A INNOVATE PERÚ, convenio N 134-FINCyT-IA-2013, al Vicerrectorado de Investigación Proyecto CON-CON 150702031.

\section{REFRERENCIAS BIBLIOGRÁFICAS}

1. Aguilar E, Rigal A. Evaluación de pinturas anticorrosivas de nanocompuestos a base de resinas epóxicas y nanoarcillas. [Tesis de maestría en Ingeniería Mecánica]. GuayaquilEcuador: Universidad de Quito; 2006.

2. Becheri A, Durr M, Lo Nostro P, Baglioni P. Synthesis and characterization of zinc oxide nanoparticles: application to textiles as UV-absorbers. J Nanopart Res. 2008; 10 : 679-689.

3. Martinello B, Rodrigues L, Michael A. Síntesis de nanopartículas de $\mathrm{ZnO}$ por el proceso SOL-GEL. Qualicer. 2009; 12(1): 1-9.

4. Yadav A, Prasad V, Kathe AA, Raj S, Yadav D, et al. Functional finishing in cotton fabrics using zinc oxide nanoparticles. Mater Sci. 2006; 29: 641-645.

5. Pérez JA, Gallego JL, Roman WS, Riascos H. Películas nanoestructuradas de óxido de zinc (ZnO). Scientia et Technica. 2008; 39: 1-15. 
6. Osama M, El Fekya, Hassanb EA, Fadelb SM, Hassanb ML. Use of ZnO nanoparticles for protecting oil paintings on paper support against dirt, fungal attack, and UV againg. J Cult Herit. 2014; 15: 165-172.

7. Hingorani S, Pillai V, Kumar P, Multani MS, Shah DO. Microemulsion mediated synthesis of Zinc-oxide Nanoparticles for Varistor Studies. Mat Res Bull. 1993; 28: 1303-1310.

8. Omri K, Najeh I, Dhahri R, El Ghoul J, El Mir L. Effects of temperature on the optical and electrical properties of $\mathrm{ZnO}$ nanoparticles synthesized by sol-gel method. Microelectron Eng. 2014; 128: 53-58.

9. Wang L, Muhammed M. Synthesis of zinc oxide nanoparticles with controlled morphology. J Mater Chem. 1999; 9: 2871-2878.

10. Davood R. Synthesis and microstructural properties of $\mathrm{ZnO}$ nanoparticles prepared by precipitation method. Renew Energ. 2013; 50: 932-937.

11. Amrut SL, Satish JS, Raghumani SN, Ahn JS, Ramchandra BP. Low temperature dielectric studies of zinc oxide $(\mathrm{ZnO})$ nanoparticles prepared by precipitation method. Adv Powder Technol. 2013; 24: 331-335.

12. Saleh R, Djaja NF. Transition-metal-dopedZnOnanoparticles: Synthesis, characterization and photocatalytic activity under UV light. Spectrochim Acta A Mol Biomol Spectrosc.. 2014; 130: 581-590.

13. Raliya R, Tarafdar JC, Mahawar H, Rajesh K, Priya G, et al. ZnO nanoparticles induced exopolysaccharide production by B. subtilis strain JCT1 for arid soil applications. Int J Biol Macromol. 2014; 65: 362-368.

14. Maryanti E, Damayanti D, Gustian I, Salprima Y. Synthesis of ZnO nanoparticles by hydrothermal method in aqueous rinds extracts of Sapindusrarak DC. Mater Lett. 2014; 118: 96-98.

15. Wu C, Qiao X, Chen J, Wang H, Tan F. A novel chemical route to prepare ZnO nanoparticles. Mater Lett. 2006; 60 (15): 1828-1832.

16. Yuanfeng C, Yuguan P, Jiyue X, Guizhen S. Surficial phase-identification and structural profiles from weathered natural pyrites: A grazing-incidence X-ray diffraction study. Appl Surf Sci. 2009; 255: 4066-4073.

17. Gilles R, Rémi L, Leroy F. Probing surface and interface morphology with Grazing Incidence Small Angle X-Ray Scattering. Surf Sci Rep. 2009; 64: 255-380.

18. Tuilier MH, Pacl MJ, Anokhin DV, Ivanov DA, Rousselot C, Thiaudière D. Nanostructured titanium and aluminium nitride coatings: study by grazing incidence $\mathrm{X}$-ray diffraction and anomalous X-ray absorption and diffraction. Thin Solid Films. 2012; 1: $1-12$.

19. Mostafaei A, Nasirpouri F. Epoxy/polyaniline $-\mathrm{ZnO}$ nanorods hybrid nanocomposite coatings: Synthesis, characterization and corrosion protection performance of conducting paints. Prog Org Coat. 2014; 77: 146- 159.

20. Ramezanzadeh B, Attar MM, Farzam M. A study on the anticorrosion performance of the epoxy-polyamide nanocomposites containing $\mathrm{ZnO}$ nanoparticles. Prog Org Coat. 2011; 72: 410-422.

21. Hongwei S, Fuchun L, Enhou H, Yinghua W. Effects of Nano Pigments on the Corrosion Resistance of Alkyd Coating. J Mater Sci Technol. 2007; 23: 551-558. 